\title{
Emission of nitrous acid from soil and biological soil crusts represents an important source of HONO in the remote atmosphere in Cyprus
}

Hannah Meusel et al.

Correspondence to: Hang Su (h.su@mpic.de) and Bettina Weber (b.weber@mpic.de)

The copyright of individual parts of the supplement might differ from the CC BY 3.0 License. 


\section{Calculations of fluxes derived by dynamic chamber measurements:}

$$
\begin{aligned}
& {[\text { HONO }] \cdot \frac{f}{A} \cdot \frac{p}{R \cdot T} \cdot M_{N}=F_{H O N O-N}} \\
& {[N O] \cdot \frac{f r}{A} \cdot \frac{p}{R \cdot T} \cdot M_{N}=F_{N O-N}}
\end{aligned}
$$

[HONO], [NO] measured mixing ratios in ppb

$\mathrm{f}=$ flow rate in $\mathrm{m}^{3} \mathrm{~s}^{-1}\left(8 \mathrm{~L} \mathrm{~min}^{-1}=1.33 \times 10^{-4} \mathrm{~m}^{3} \mathrm{~s}^{-1}\right)$

$\mathrm{A}=$ surface of sample in $\mathrm{m}^{2}\left(0.00238 \mathrm{~m}^{2}\right)$

$\mathrm{p}=$ pressure in $\mathrm{Pa}$

$\mathrm{R}=$ ideal gas constant $\left(8.31 \mathrm{~J} \mathrm{~mol}^{-1} \mathrm{~K}^{-1}\right)$

$\mathrm{T}=$ temperature in $\mathrm{K}(298 \mathrm{~K})$

$\mathrm{M}_{\mathrm{N}}=$ molar weight of $\mathrm{N}\left(14 \mathrm{~g} \mathrm{~mol}^{-1}\right)$

$\mathrm{F}_{\mathrm{HONO}-\mathrm{N}}, \mathrm{F}_{\mathrm{NO}-\mathrm{N}}=$ fluxes of HONO-N and NO-N in $\mathrm{ng} \mathrm{m}^{-2} \mathrm{~s}^{-1}$ 
Table S1: Overview over soil and biocrust samples including nutrient and chlorophyll analyses and HONO and NO emission fluxes. $(<\mathrm{LOD}=$ below limits of detection $)$

\begin{tabular}{|c|c|c|c|c|c|c|c|c|c|c|c|c|}
\hline \multicolumn{2}{|l|}{ sample type } & \multirow{2}{*}{$\begin{array}{l}\mathrm{NO}_{2}{ }^{-}-\mathrm{N} \\
0.126\end{array}$} & $\begin{array}{l}\mathrm{NO}_{3}^{-}-\mathrm{N} \\
\mathrm{mg} \mathrm{kg}^{-1}\end{array}$ & $\mathrm{NH}_{4}^{+}-\mathrm{N}$ & \multicolumn{2}{|c|}{$\mathrm{mg} \mathrm{m}^{-2} \mathrm{chl}_{\mathrm{a}-\mathrm{b}}$} & \multicolumn{2}{|c|}{$\begin{array}{c}\mathrm{HONO}_{\max } \quad \mathrm{NO}_{\max } \\
\mathrm{ng}(\mathrm{N}) \mathrm{m}^{-2} \mathrm{~s}^{-1}\end{array}$} & \multicolumn{2}{|c|}{$\begin{array}{c}\mathrm{HONO}_{\text {int }} \quad \mathrm{NO}_{\text {int }} \\
\mu \mathrm{g}(\mathrm{N}) \mathrm{m}^{-2}\end{array}$} & \multicolumn{2}{|c|}{ HONO/NO } \\
\hline Bare soil & 1 & & 0.723 & 2.017 & 17.45 & 8.61 & 89.12 & 53.70 & 465.49 & 341.45 & 1.66 & 1.36 \\
\hline Bare soil & 2 & 0.574 & 2.450 & 1.325 & 18.66 & 6.84 & 263.80 & 120.99 & 1899.9 & 1544.8 & 2.18 & 1.23 \\
\hline Bare soil & 3 & 0.501 & 6.478 & 6.509 & 31.71 & 13.93 & 173.32 & 101.83 & 1510.4 & 1621.3 & 1.70 & 0.93 \\
\hline Dark BSC & 1 & $<$ LOD & $<$ LOD & 1.906 & 40.80 & 27.48 & 1.29 & 1.73 & 9.45 & 17.1 & 0.74 & 0.55 \\
\hline Dark BSC & 2 & $<$ LOD & $<$ LOD & 1.873 & 32.13 & 18.61 & 3.08 & 2.90 & 16.42 & 25.49 & 1.06 & 0.64 \\
\hline Dark BSC & 3 & $<$ LOD & 0.050 & 1.365 & 30.42 & 15.52 & 4.69 & 6.64 & 36.28 & 63.53 & 0.71 & 0.57 \\
\hline Dark BSC & 4 & 0.265 & 3.549 & 3.159 & 95.66 & 66.01 & 43.15 & 35.86 & 337.45 & 359.56 & 1.20 & 0.94 \\
\hline Dark BSC & 5 & 0.113 & 0.582 & 2.061 & 21.65 & 11.26 & 83.43 & 85.1 & 443.86 & 712.1 & 0.98 & 0.62 \\
\hline Light BSC & 1 & $<$ LOD & $<L O D$ & 0.626 & 12.71 & 6.05 & 1.28 & 1.74 & 8.61 & 14.84 & 0.73 & 0.58 \\
\hline Light BSC & 2 & $<$ LOD & $<L O D$ & 0.587 & 16.34 & 7.72 & 12.35 & 11.44 & 61.48 & 66.07 & 1.08 & 0.93 \\
\hline Light BSC & 3 & 0.267 & 4.015 & 22.209 & 24.60 & 11.00 & 96.53 & 95.22 & 540.77 & 592.28 & 1.01 & 0.91 \\
\hline Light BSC & 4 & 0.119 & 0.819 & 1.478 & 18.09 & 8.31 & 83.89 & 67.5 & 475.72 & 481.0 & 1.24 & 0.99 \\
\hline Chlorolichen BSC I & 1 & $<L O D$ & $<L O D$ & 0.085 & 61.39 & 37.48 & 0.63 & 0.83 & 3.73 & 6.03 & 0.76 & 0.62 \\
\hline Chlorolichen BSC I & 2 & $<L O D$ & $<L O D$ & $<L O D$ & 84.12 & 58.64 & 2.45 & 2.62 & 12.02 & 15.73 & 0.93 & 0.76 \\
\hline Chlorolichen BSC I & 3 & $<L O D$ & $<L O D$ & 0.829 & 107.59 & 74.85 & 1.24 & 2.03 & 10.50 & 24.54 & 0.61 & 0.43 \\
\hline Chlorolichen BSC II & 1 & $<L O D$ & $<L O D$ & 0.187 & 24.75 & 14.18 & 1.69 & 1.88 & 15.23 & 15.32 & 0.90 & 0.99 \\
\hline Chlorolichen BSC II & 2 & 0.011 & 0.116 & 2.460 & 10.58 & 10.58 & 7.98 & 8.40 & 54.53 & 63.25 & 0.95 & 0.86 \\
\hline Chlorolichen BSC II & 3 & 0.074 & 0.916 & 0.982 & 21.73 & 12.29 & 9.65 & 9.88 & 94.72 & 103.62 & 0.98 & 0.91 \\
\hline Chlorolichen BSC II & 4 & 0.017 & 0.128 & 2.062 & 17.97 & 9.50 & 19.97 & 15.83 & 110.68 & 95.15 & 1.26 & 1.16 \\
\hline Chlorolichen BSC II & 5 & $<L O D$ & 0.513 & 3.894 & 37.46 & 22.65 & 4.27 & 4.43 & 35.14 & 49.83 & 0.96 & 0.71 \\
\hline Chlorolichen BSC II & 6 & $<\mathrm{LOD}$ & $<$ LOD & 0.585 & 17.43 & 9.71 & 1.52 & 1.54 & 7.87 & 11.60 & 0.98 & 0.68 \\
\hline Moss BSC & 1 & 0.071 & $<$ LOD & 2.048 & 48.93 & 27.82 & 12.68 & 13.44 & 104.08 & 148.62 & 0.94 & 0.70 \\
\hline Moss BSC & 2 & $<\mathrm{LOD}$ & $<\mathrm{LOD}$ & 0.306 & 83.63 & 54.53 & 4.34 & 5.79 & 40.07 & 57.66 & 0.75 & 0.69 \\
\hline Moss BSC & 3 & 0.030 & $<\mathrm{LOD}$ & 0.763 & 211.31 & 144.21 & 6.78 & 8.87 & 62.54 & 89.10 & 0.77 & 0.70 \\
\hline Moss BSC & 4 & $<\mathrm{LOD}$ & 0.029 & 5.164 & 169.64 & 123.26 & 3.49 & 3.65 & 16.61 & 19.58 & 0.96 & 0.85 \\
\hline
\end{tabular}




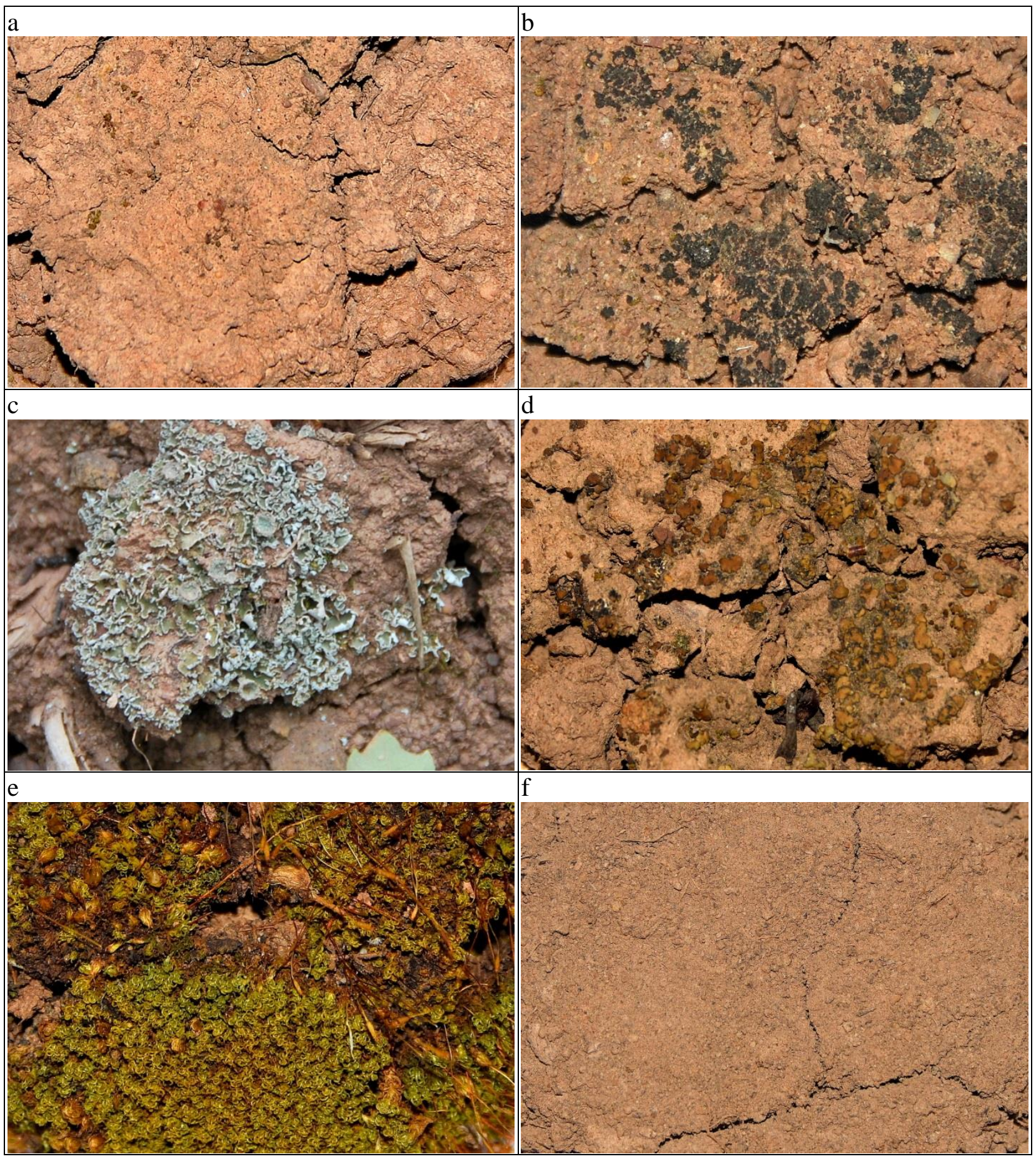

Fig. S1: Pictures of local biocrusts: a) light cyanobacteria-dominated biocrust, b) dark cyanobacteriadominated biocrust with Collema sp. as dominating cyanolichen species, c) chlorolichen-dominated biocrust with Cladonia sp. as dominating lichen species, type I, d) chlorolichen-dominated biocrust with Placidium sp. as dominating lichen species, type II, e) moss-dominated biocrust with Trichostomum crispulum as dominating moss species, and f) bare soil. 

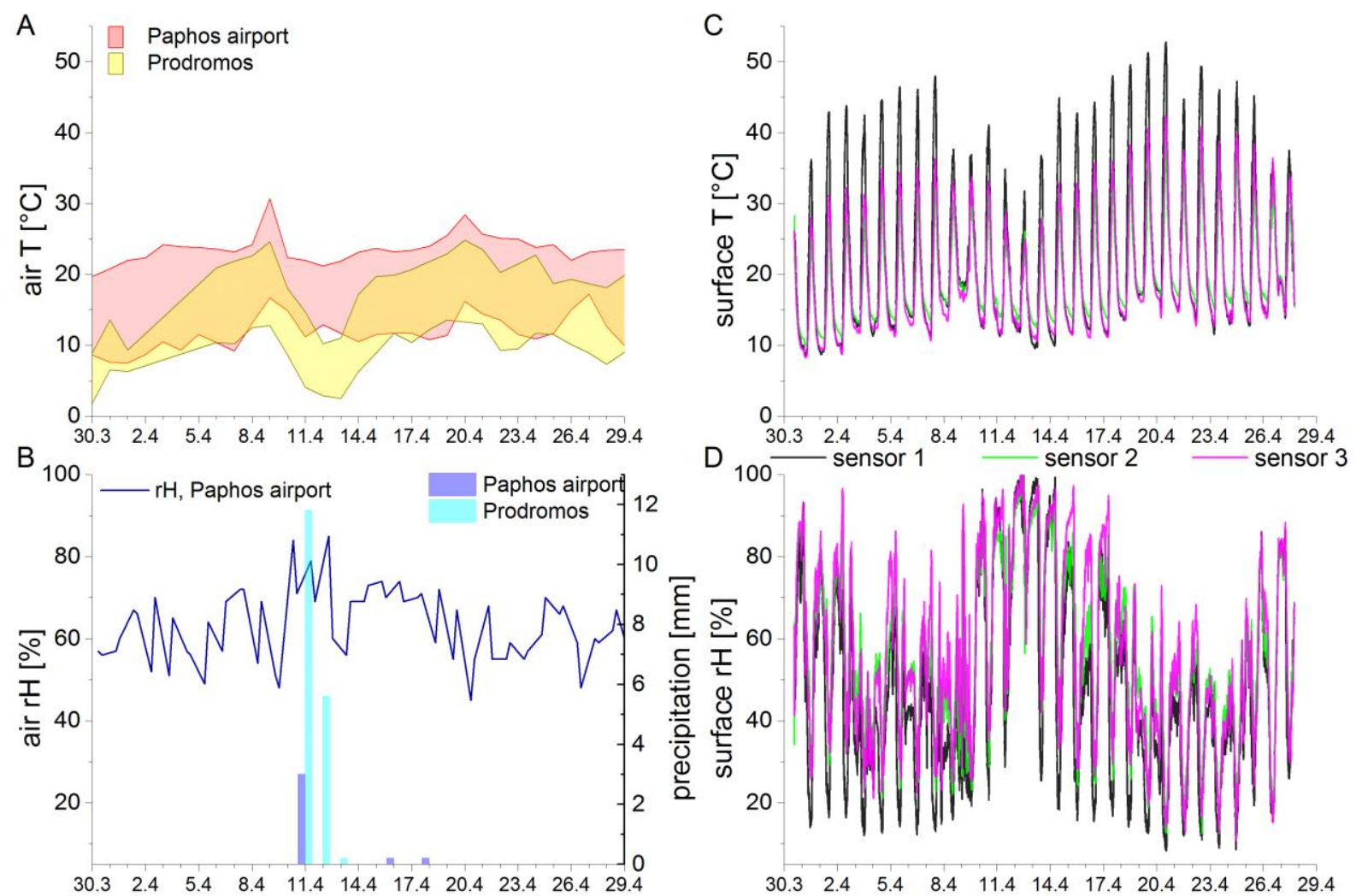

Fig. S2: Climatic conditions of air and soil during April 2016, about one month before samples were taken. Atmospheric data was adopted from the Department of Meteorology, Cyprus. Minimum and maximum air temperatures (A) of one day at both sites are presented by red and yellow shaded areas. Air-rH data (B; dark blue line, left axis) were only available for Paphos airport, representing values at 8:00 and 13:00 local time. Precipitation data at Paphos airport and Prodromos (B; blue bars, right axis) show the daily rainfall. Surface temperature and $\mathrm{rH}$ are shown on the right side $(\mathrm{C}, \mathrm{D})$. The time resolution is $5 \mathrm{~min}$. The variations between sensors arise from 3 different locations/surface (bare soil, next to rock, under shrubs).

(http://www.moa.gov.cy/moa/ms/ms.nsf/DMLmeteo_reports_en/DMLmeteo_reports_en?OpenDocument) 


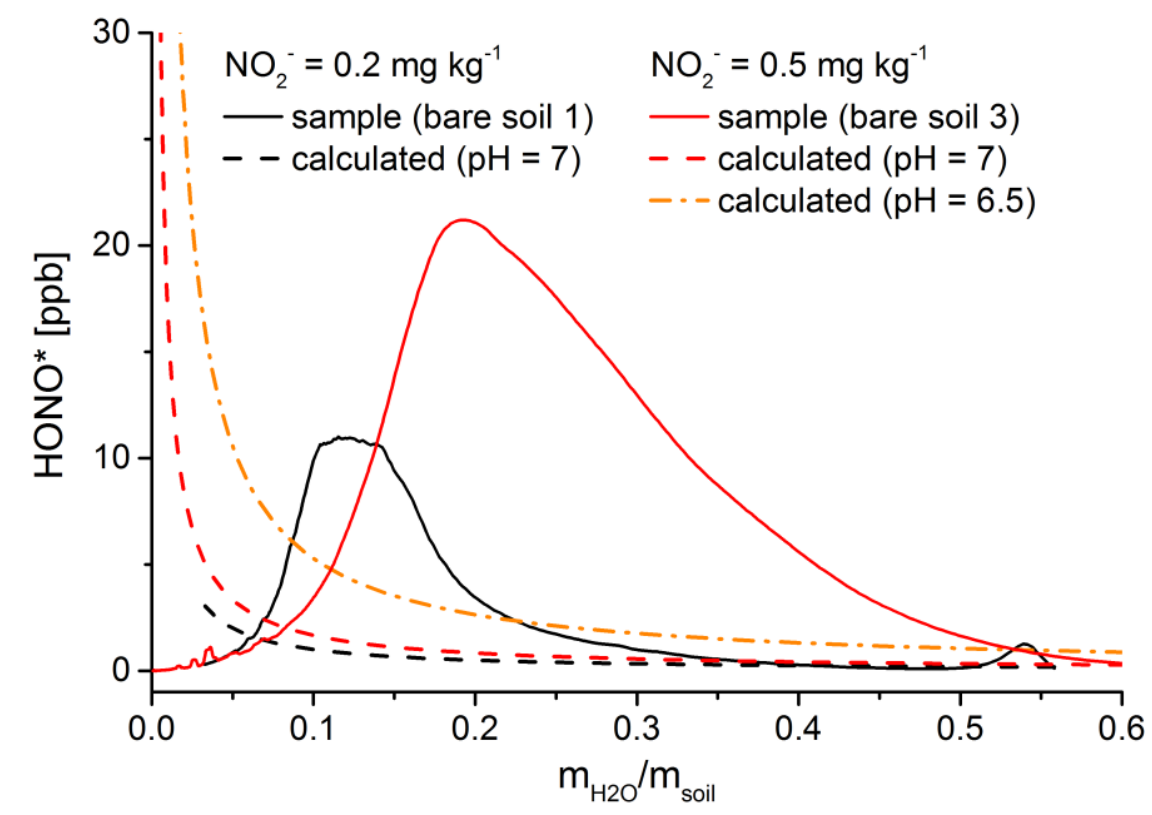

Fig. S3: Calculated [HONO]* for two different $\mathrm{NO}_{2}^{-}$concentrations at $\mathrm{pH} 7$ and pH 6.5 (dashed lines) in comparison with measured [HONO]* for two samples with similar $\mathrm{NO}_{2}^{-}$content (solid lines) vs the gravimetric soil water content. 